\title{
Dynamic compaction machine boom structure dynamics research
}

\author{
Yuming Sun ${ }^{a}$, Ning Sun ${ }^{b}$ \\ School of Mechanical and Electrical Engineering, Guilin University of Electronic Technology, Guilin \\ 541004, China \\ asunyuming1@126.com, bsunning@guet.edu.cn
}

Keywords: Boom, suddenly unloading, transient dynamics analysis.

\begin{abstract}
According to the structure of dynamic compaction machine boom, under the condition of each parameter, lists the boom in sudden unloading conditions of motion differential equations, using the finite element software workbench analysis rammer suddenly unloaded and the changes of stress with time to find the biggest boom stress position, analysis of the reliability of the boom frame structure performance, for dynamic compaction machine boom frame of research and optimization to provide important theoretical basis.
\end{abstract}

\section{Introduction}

Dynamic compaction method of dynamic compaction method to a certain height after free fall, tamping soil foundation with shock and vibration, make its rapid consolidation method. Since its birth, the method for its economic and feasible, effective, simple equipment, construction is convenient, save material, easy to control quality, wide applicable scope, short construction period and other advantages In construction process, due to the boom deformation and flexible luffing system can lay aside, after the static equilibrium, the rebound and vibration and dynamic response, especially the rammer in sudden unloading operation conditions, it is the boom has a great impact on the stability and the stability of the whole machine, may lead to major accidents[1]. therefore, the research of sudden unloading condition of dynamic compaction machine boom has important theoretical value and engineering significance in QUY600 caterpillar dynamic compaction machine as the research object, through the establishment of the boom frame structure under sudden unloading conditions of dynamic model, using the finite element method for dynamic analysis of the boom is analyzed with the maximum under the condition of the working load stress position, is also the most weak link of boom system, for the improvement of the structure of the dynamic compaction boom after powerful reference basis.

\section{Model analysis}

After the lifting of the stationary state, the stress of the dynamic compaction machine boom schematic fig.1.F as the pull force; $\mathrm{P}$ for The force anti-roll bar; $\mathrm{G}$ for boom weight; $\mathrm{T}$ is the weight of rammer and hook $\alpha$ for boom elevation; $\beta$ to pull on the rope tension and arm center axis angle; $\theta$ for anti-roll bar on the force and the Angle of the center of a boom. $n$ is pulley block ratio. Dynamic compaction machine parameters are as follows: the weight of $\mathrm{m} 1=25 \mathrm{t}$, hook quality $\mathrm{m} 2=1.7 \mathrm{t}$, the quality of the arm $\mathrm{m} 3=5.3$, lifting pulley block ratio $\mathrm{n}=2$. Boom 24 meters long According to the torque balance equation:

$$
\begin{aligned}
& (\mathrm{T}+0.5 \mathrm{G})^{*} \cos \alpha-\mathrm{F}^{*} \sin \beta+0.4 \mathrm{P} * \sin \theta=0 \\
& \mathrm{~F}=\mathrm{T} / \mathrm{n}
\end{aligned}
$$

According to the equation can be obtained $\mathrm{P}=149000$ N.In order to make the model more reasonable and more convenient in calculation, needs the actual boom in simplified place. To simplify the model after decommissioning should follow principles: (1) Model can allow it stands all face to the arm structure characteristics; (2) The model load should work with the boom frame outside loading phase.(3) The model of border area should keep and boom the actual work a cause.Chord and 
web member choose linear beam element BEAM188, choose SHELL63 goose head and its reinforcement plate[2].

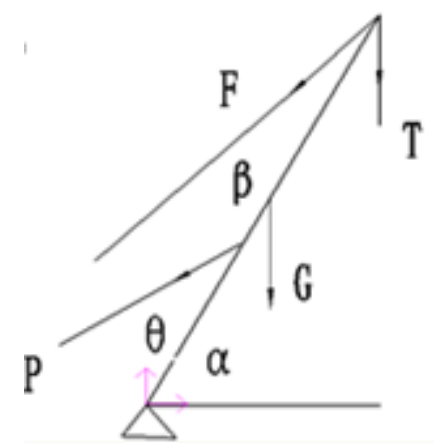

Fig.1 The stress of the boom structure figure

Table1 Boom section size and material properties

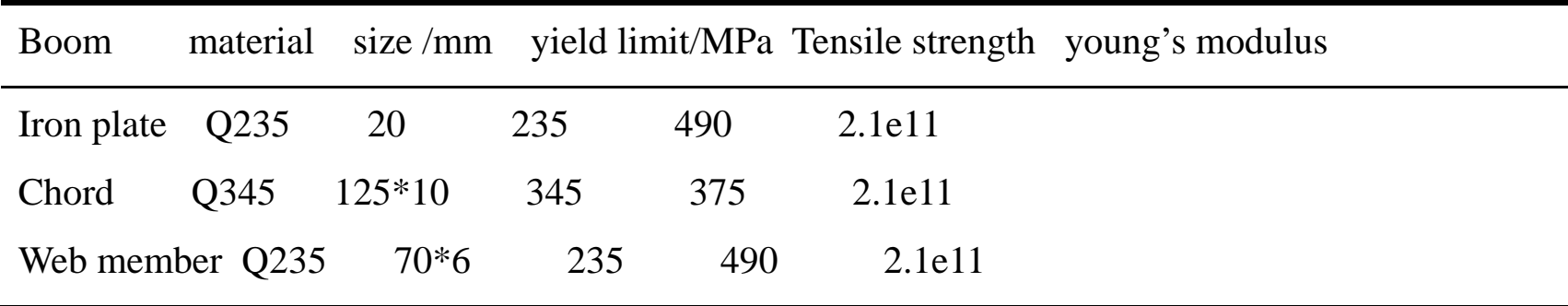

Because dynamic compaction machine haven't design specifications, design reference to crane design specification, now its tensile, compression, bending allowable Stress can be divided into the following two conditions to calculate.

$$
\begin{aligned}
& \frac{\partial \mathrm{s}}{\partial b}<0.7, \quad[\partial]=\frac{\partial \mathrm{s}}{n} \\
& \frac{\partial \mathrm{s}}{\partial b} \geq 0.7, \quad[\partial]=\frac{0.5 \partial \mathrm{s}+0.35 \partial b}{n}
\end{aligned}
$$

According to crane design specification chosen as 1.34.The basic allowable stress respectively calculate: main arm main chord allowable stress 257MPa, Web member allowable stress 175MPa. According to the analysis of the figure 3 safety coefficient is much larger than 1.34 , so the structure is very safety

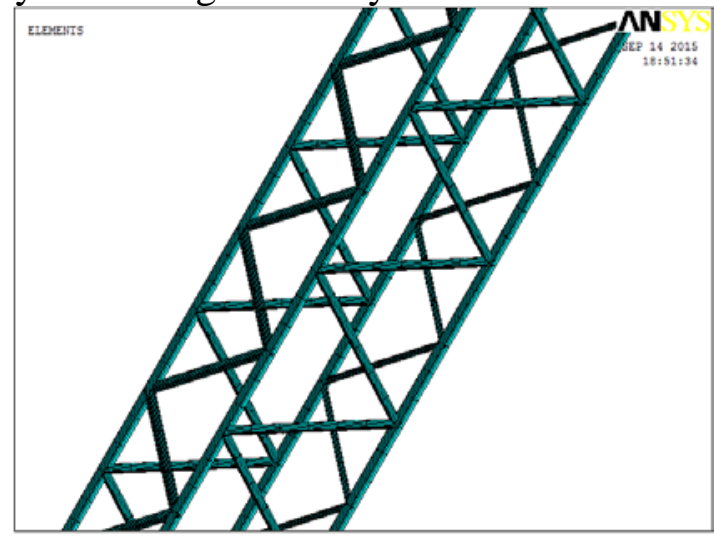

Fig.2 Part of the Mesh model

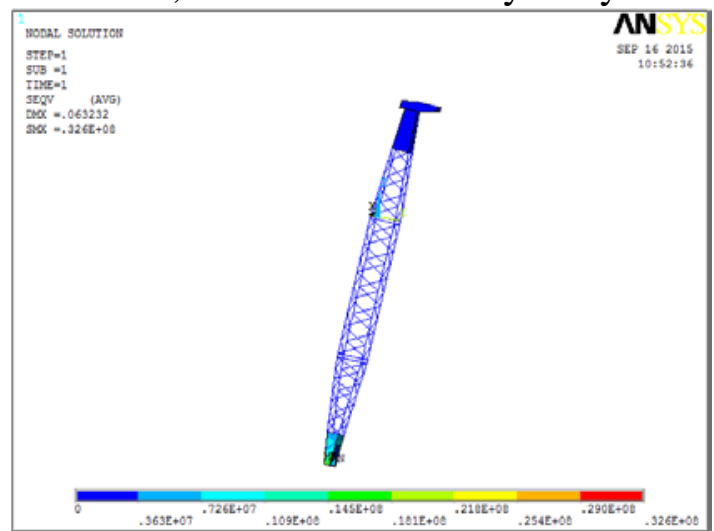

Fig.3 Boom statics analysis

By the vibration theory, the low order natural frequency of the system have a larger impact on the dynamic response and high order natural frequency is less affected. So only need to analyze the many degree of freedom system Low order natural frequency can reflect the dynamic characteristics of system, so this article only extracted first six order modal. According to the analysis of the front arm load, In the arm frame applied load and constraints, the sway bar, pull on the rope, lifting load is exerted on the arm of the corresponding node, The modal results below. 
Table 2 Before the boom six natural frequencies

\begin{tabular}{lclccc}
\hline Set & Time/Freq & Load Step & Substep & Cumulative \\
\hline 1 & 1.0541 & 1 & 1 & 1 & \\
2 & 1.5932 & 1 & 2 & 2 & \\
3 & 7.7286 & 1 & 3 & 3 & \\
4 & 11.623 & 1 & 4 & 4 & \\
5 & 13.612 & 1 & 5 & 5 & \\
6 & 18.606 & 1 & 6 & 6 & \\
\hline
\end{tabular}

We can see from the modal figure: Each order the main vibration mode mainly for horizontal or vertical direction vibration and torsional pendulum. In the middle of the $13 \mathrm{~Hz}$ natural frequency boom arm section prone to bending deformation, So, when the boom structure design should strengthen this part of the strength.


Fig.5 The Five order modal

\section{Transient dynamic analysis}

The transient dynamic analysis is used to determine the change over time of load of the structure of the dynamic response of a method. We can use the transient dynamics analysis to determine structure under static load, the transient load and harmonic load under the action of an arbitrary combination of stress, strain and displacement change over time and force. Load and time correlation makes the inertia force and damping effect is more important. Because of the dynamic compaction machine boom suddenly unloading situation, the effect of inertia force is very significant, so the reliability of the transient analysis to determine his is very necessary. According to the time step in ANSYS transient dynamics analysis principle to determine the time step.

$$
T=\frac{1}{20 * f}
$$


According to boom finite element model, using the Block Lanczos method modal analysis and calculation, get the boom frame structure in the range of in-plane vibration frequency at a rate of $13.612 \mathrm{~Hz}$, therefore, the analysis time interval for $0.0036 \mathrm{~s}[3,4]$. The method of calculating the transient dynamics analysis is full in this paper.

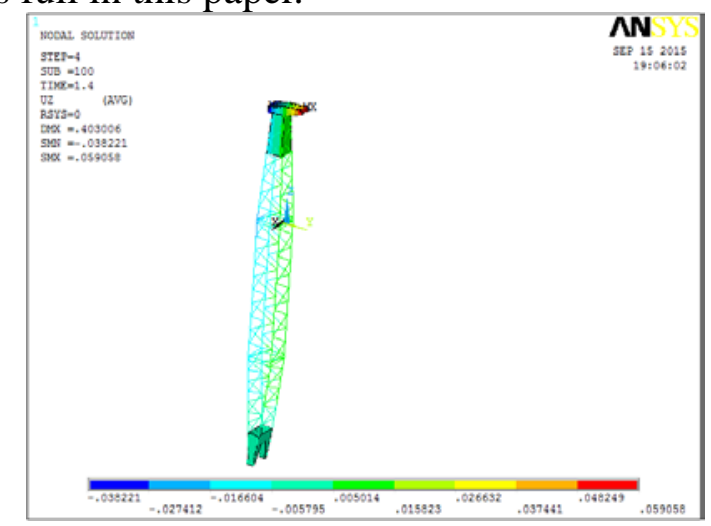

Fig.6 The displacement vector nephogram



Fig.7 The node corresponding response curve

After unloading dynamic compaction machine, the boom can produce the spring back, actually in the middle section bending changes will be the most obvious. Using ANSYS analysis to completely conform to the actual situation, this method is correct. Boom after unloading rebound due to bending displacement change is the biggest node will produce large displacement, then can produce small bending because of the weight of the hook, this also with the analysis of the figure 7 perfectly.

\section{Conclusion}

From the previous analysis safety coefficient is very large, so it is necessary for structural optimization design, The result of dynamics analysis enables a designer can predict the stability of the boom performance, in order to optimize the arm to provide certain reference for structure design, and also provide reference for other series analysis of the boom.

\section{Reference}

[1]Xilang Wang, Weihou Shui. The development and application of dynamic compaction machine [J]. Engineering machinery, 2004.6 p.35

[2]Wenzhi Zhang, Qingkai Han, Hong Liu, mechanical structure finite element analysis, etc. [M] Harbin industrial university press, 2006:P.7-9

[3]Haifang Li. Crane boom system coupled transient dynamics simulation analysis [D].Wuhan: wuhan university of technology, 2010.5

[4]Pengming Lv, Ding Zhi. Transient dynamic analysis of the main girder structure [J]. Journal of chang 'an university (natural science edition), 2005, 25 p.80-83 